\title{
Review Paper: \\ Strategies for the Prevention of Ventilator-associated Pneumonia in the Intensive Care Units: A Review
}

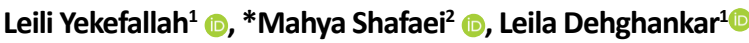

1. Social Determinants of Health Research Center, Institute for Prevention of Non-Communicable Diseases, School of Nursing \& Midwifery, Qazvin University of Medical Sciences, Qazvin, Iran.

2. Department of Nursing, School of Nursing and Midwifery, Qazvin University of Medical Sciences, Qazvin, Iran.

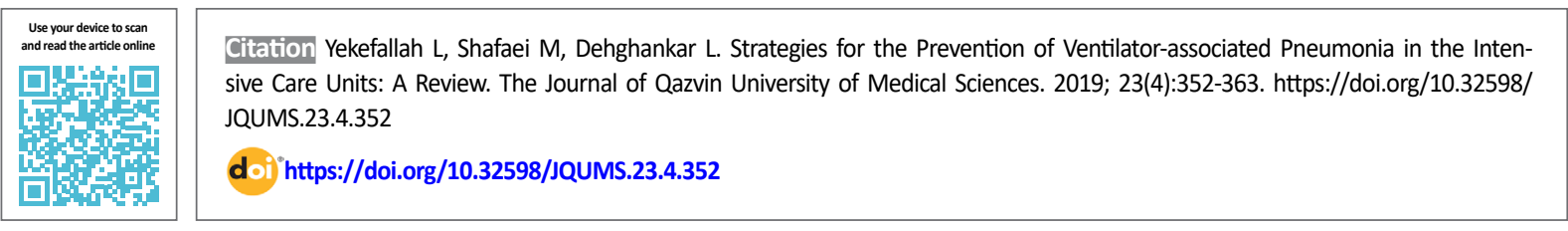

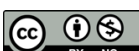

Received: 23 Apr 2019

Accepted: 21 Aug 2019

Available Online: 01 Oct 2019

Keywords:

Ventilator-associated pneumonia, Intensive Care Units, review

\section{A B STRACT}

Ventilator-Associated Pneumonia (VAP) accounts for $80-90 \%$ of hospital-acquired pneumonia cases in Intensive Care Units (ICUs). VAP occurs 48-72 hours after intubation, and is observed in $27-29 \%$ of patients with endotracheal tube, and its risk increases with the increase in the duration of mechanical ventilation. In this review study, papers published from 1996-2018 were used to investigate strategies for preventing VAP in ICU patients. For this purpose, English articles with full text available on ProQuest, SID, ScienceDirect, Google Scholar and Scopus databases were searched online using the keywords related to VAP and chest physiotherapy used in the title or abstract. A total of 50 articles (from 10 different countries) covering both qualitative and quantitative studies in Persian and English conducted the last 19 years (since 1999) were selected. Those which were not a review/research paper and their full text were unavailable were excluded from the review. Among nosocomial infections, VAP is the most common cause of death, and the leading cause of death in ICUs. Physical strategies with a significant effect on the reduction of VAP included: Orotracheal intubation, closed endotracheal suctioning, use of humidifier and moisturizer, and chest physiotherapy. Effective positional strategies were: the use of kinetic beds, semi-recumbent position (45 degrees), and prone position. Effective medication strategy is the use of topical and intravenous antibiotic prophylaxis.

\section{Extended Abstract}

\section{Introduction}

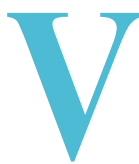

entilator-associated pneumonia (VAP) accounts for $80-90 \%$ of hospital-acquired pneumonia cases in Intensive Care Units (ICUs). VAP occurs 48-72 hours after intubation, and is observed in $27-29 \%$ of patients with endotracheal tube, and its risk increases with the increase in the duration of mechanical ventilation [8]. Among nosocomial infections, VAP is the most common cause of death and the leading cause of death in ICUs. VAP accounts for $20-70 \%$ of hospital deaths [3]. Nosocomial pneumonia is the second most common nosocomial infection with bacterial origin. Hospital pneumonia and VAP increase the healthcare cost and the length of hospital stay [12].

VAP is classified into two types: Early onset (up to 96 hours after mechanical ventilation) and late onset (more than 96 hours after mechanical ventilation) [4]. There are many factors that can cause this type of infection in patients undergoing mechanical ventilation. The accumu-

\section{* Corresponding Author:}

Mahya Shafaei

Address: Department of Nursing, School of Nursing and Midwifery, Qazvin University of Medical Sciences, Qazvin, Iran

Tel: +98 (28) 33338034

E-Mail: shafaiimahya@gmail.com 
lation of pharyngeal secretions behind the endotracheal tube cuff causes unclear aspiration, loss of cough reflex, airway cilia dysfunction, inactivity and being in a long sleeping position, the need to use suction to discharge secretions and the presence of antibiotic-resistant bacteria transmitted to patients via the contaminated hands of healthcare staff [6].

The VAP is characterized by clinical symptoms such as fever, purulent sputum, and laboratory symptoms such as new lobular filtration in chest images, positive sputum culture, and aspirated tracheal secretions [7]. The risk of VAP occurrence during the first 5 days of mechanical ventilation is $3 \%$ per day, $2 \%$ in the next $5-10$ days and $1 \%$ per day after 10 days [4]. The high rate of VAP prevalence in Iran is observed in patients with respiratory distress syndrome [9].

Many strategies have been proposed to prevent and minimize this complication. Adjusting the bed slope, regulating the pressure in the endotracheal tube cuff and removal of oral secretion are some of these methods. Some scholars have suggested that raising nurses' awareness and their compliance with the ICU guidelines can have a significant impact on reducing the incidence of VAP $[17-19,21,20]$. Some studies have also indicated that different nursing interventions such as hand washing and closed suctioning can reduce the incidence of VAP [22, 23]. Considering the importance of controlling and preventing VAP in ICUs, the present study aimed to review the approaches to VAP prevention in these hospital units.

\section{Materials and Methods}

In this review study conducted in 2018, articles indexed in ProQuest, SID, ScienceDirect, Google Scholar and Scopus databases were used. Papers published from 1996-2018 related to both quantitative and qualitative studies, published in Persian and English with available full-text related to the approach to VAP prevention were included in the study. The criteria for selecting web portals for the search were having "ac" or "edu" domain in addition to its relation to the subject. They were searched using the google search engine.

After checking the title of papers, they were then checked in terms of abstract relevance to the study purpose. Exclusion criteria were those whose method was unclear and their full texts were unavailable. In this regard, first one of the researchers (master in nursing) examined the articles, conducted searches, and entry/exit criteria according to the title and abstract. Other researcher was responsible for writing and validating the resources. After excluding the articles that did not meet the inclusion criteria, 49 from 96 articles were selected and their full texts were reviewed. Then, their results were extracted and examined according to the study factors.

\section{Conclusion}

VAP is the most common cause of death among nosocomial infections and is the leading cause of death in ICUs. Endotracheal tube and poor oral hygiene can lead to VAP. The Center for Disease Control and Prevention in US has proposed a comprehensive oral-hygiene program (including antiseptic agents) for patients at risk of VAP [31]. Hospital pneumonia is associated with dental plaque and pharyngeal colonization [32, 33]. The American Association of Critical Care Nurses recommends tooth brushing twice a day, oral swab every 2-4 hours, and regular suctioning of oral cavity to reduce the risk of VAP occurrence.

The studies that reported toothbrushes as a risk factor for VAP, have attributed it to the decline of microbial agents following tooth brushing and their transmission to the lung [40]. Pharmacological interventions also have a great impact on reducing pharyngeal microbes and thus preventing VAP. Craven et al. reported that purposive use of antibiotics significantly reduced the prevalence of Ventilator-Associated Tracheobronchitis (VAT) and better prevention results were observed in patients by using antibiotics [35]. One study showed that twice-daily chest physiotherapy significantly reduced the incidence of VAP compared to controls. This physiotherapy also reduced the mortality rate in these patients [41].

Effective suctioning also plays a very effective role in preventing VAP, especially its early onset type. Another study reported that the rate of VAP was higher in closed suctioning system than in open suctioning system [4446]. Shaking is another technique performed manually; however, its effect on reducing VAP incidence has not yet been proven, but it is widely used to help clear airway secretions. Although VAP is a common nosocomial infection and can increase the cost of treatment and length of hospital stay, it can be prevented or minimized by simple and applicable strategies. Physical strategies include orotracheal intubation, closed endotracheal suctioning, use of humidifier, and chest physiotherapy; positional strategies are the use of kinetic beds, semi-recumbent positioning $\left(45^{\circ}\right)$, prone position; and medication strategy is the use of topical and intravenous antibiotic prophylaxis. These methods have a significant effect on reducing the VAP prevalence. 


\section{Ethical Considerations}

Compliance with ethical guidelines

This review article does not require ethical observation concerning the type of study.

Funding

This research did not receive any specific grant from funding agencies in the public, commercial, or not-forprofit sectors.

Authors' contributions

Investigation, resources, data analysis, editing, and draft preparation: Mahya Shafaei; Investigation: Leili Yekefalla, Leila Dehghankar; Resources, writing and editing: Leili Yekefallah; Editing by Leila Dehghankar.

Conflicts of interest

The authors declared no conflict of interest. 


\section{راهكارهاى ييشكَيرى از بروز هنومونى ناشى از ونتيلاتور در بخش مراقبتهاى ويؤه: يك مطالعه مرورى

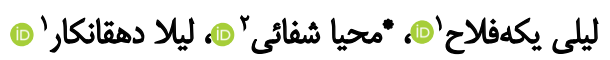

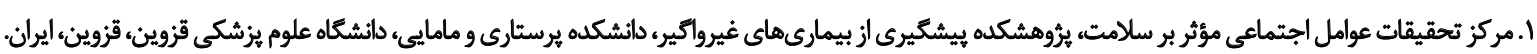

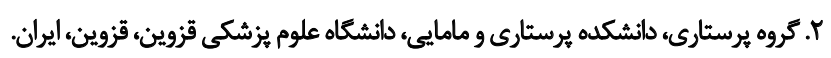

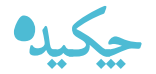

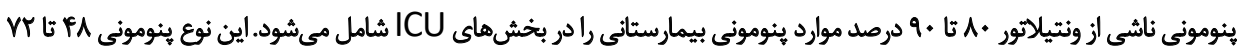

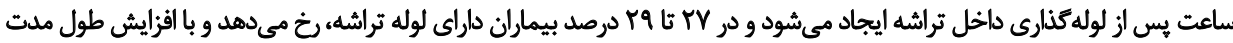

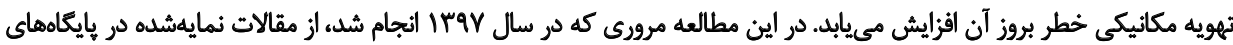

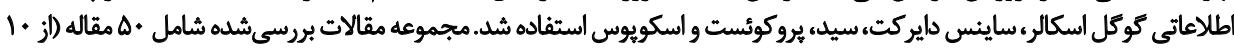

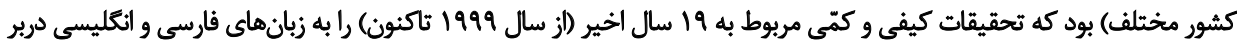

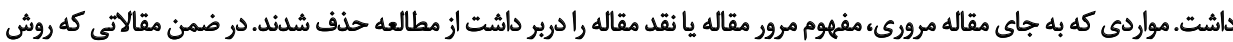

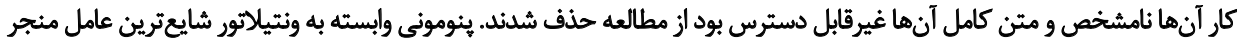

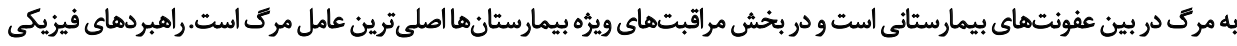

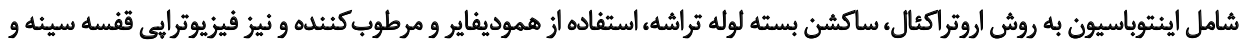

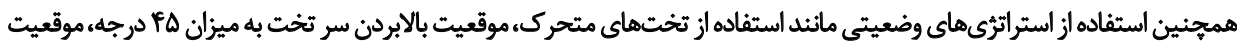

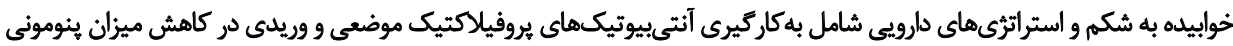
ناشى از ونتيلاتور تأثير قابل توجهي دارئ

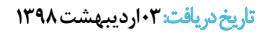

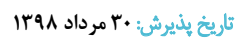

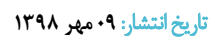

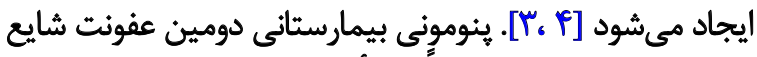

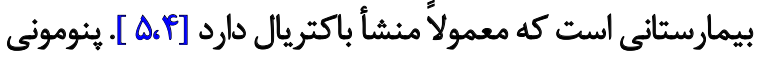

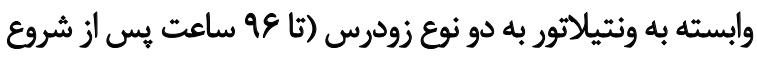

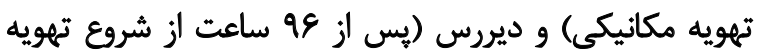

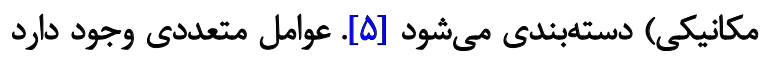

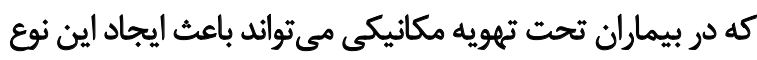

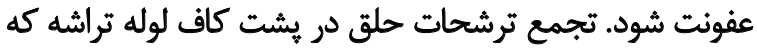

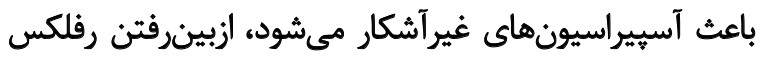

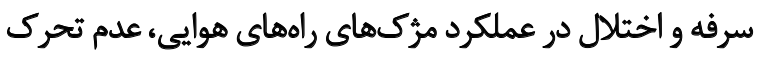

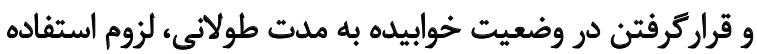

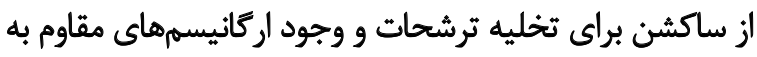

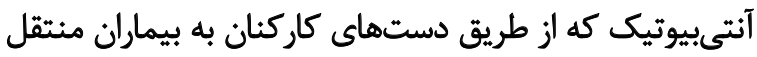

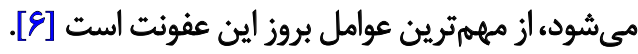
تشخيص ينومونى ناشى از تهويه مكانيكى با علائم بالينى از

لولهكذارى داخل تراشه و اتصال بيماران به دستكاه تهويه

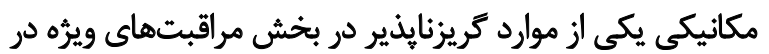

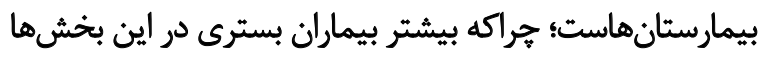

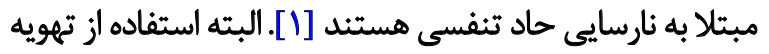

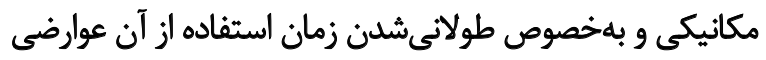

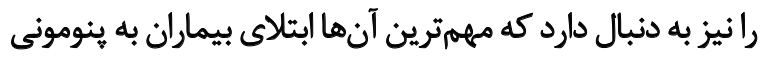

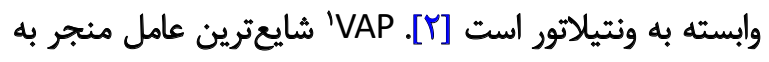

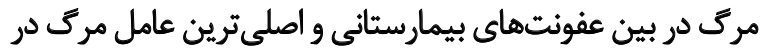

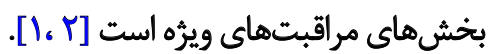

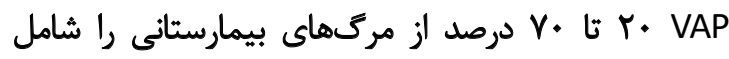

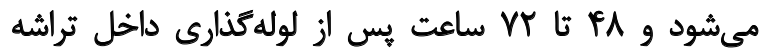

1. Ventilator Associated Pneumonia (VAP)

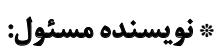

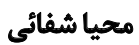

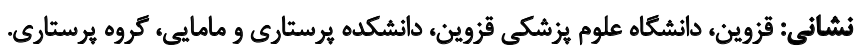

$$
\begin{aligned}
& \text { تلفن: } \\
& \text { رايانامه: shafaiimahya@gmail.com }
\end{aligned}
$$




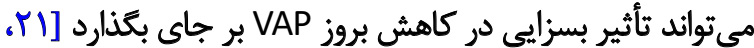

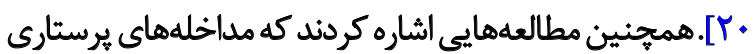

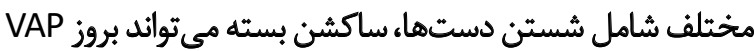

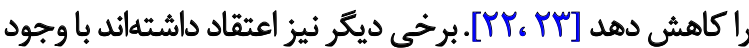

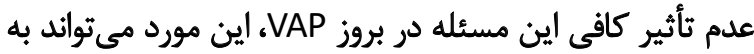

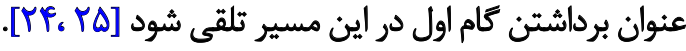
كوكانور و همكاران طى مطالعهاى كزارش كردند زمانى كه به به

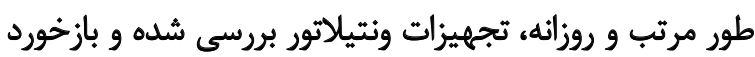

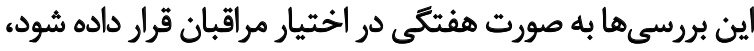

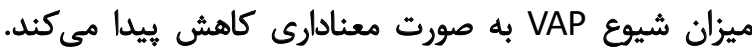

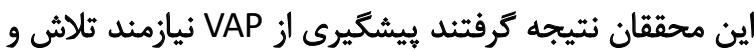

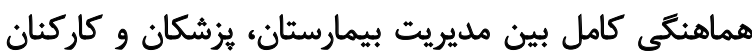

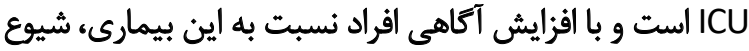

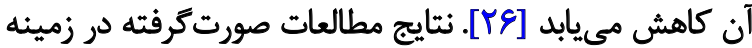

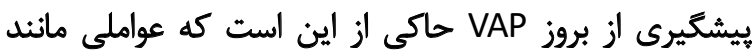

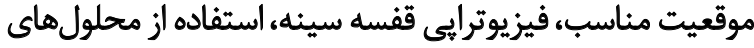

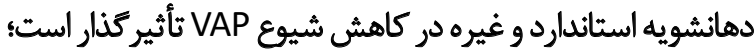

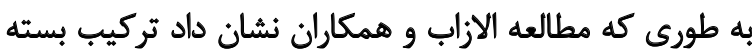

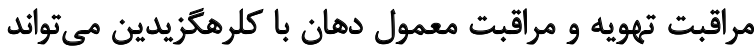

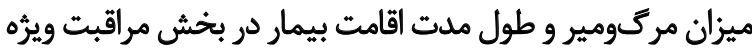

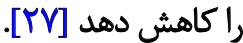

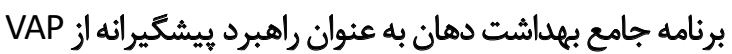

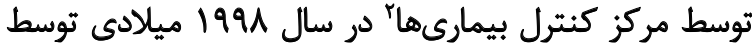

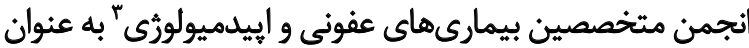

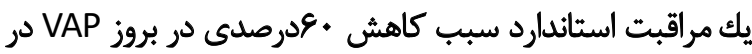

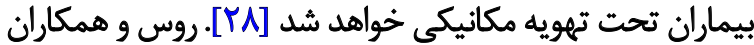

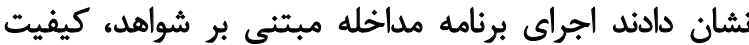

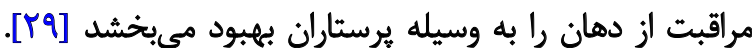

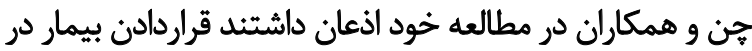

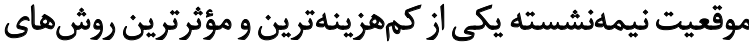

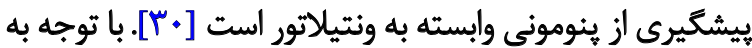

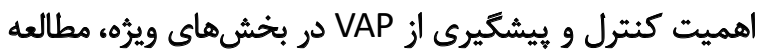

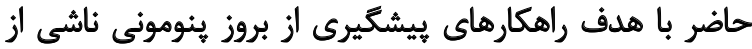

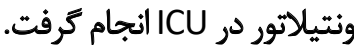

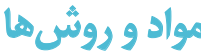

با توجه به موضوع مطالعه، مقالات تحقيقى و مرورىاي كه از إز إياز

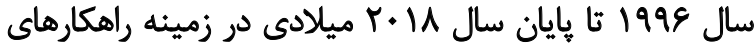

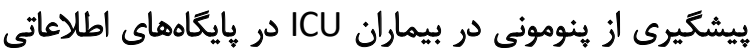

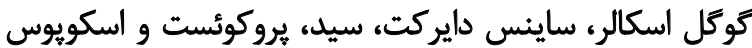

\section{Centers for control disease (CDC)}

3. Association for professionals in infection control and epidemiology (APIC)
قبيل تب، خلط جركى و علاثم آزمايشكاهى از قبيل فيلتراسيون

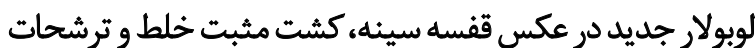

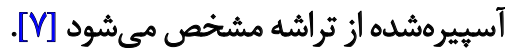

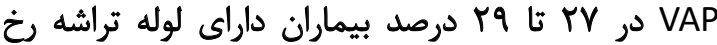

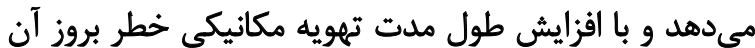

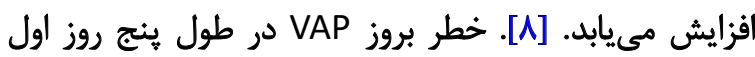

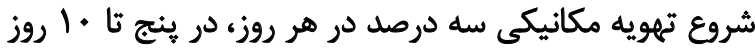

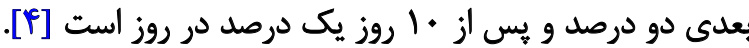

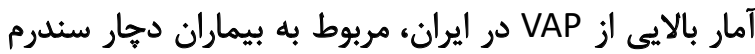

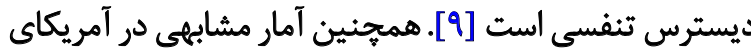

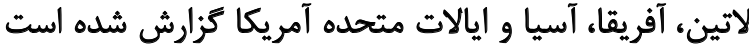

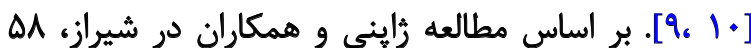

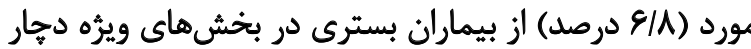

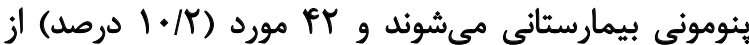

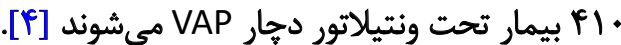

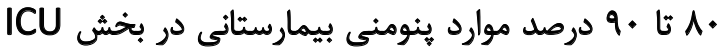

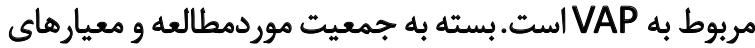

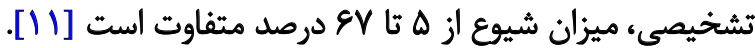

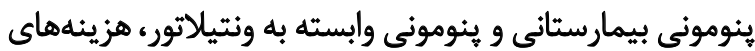

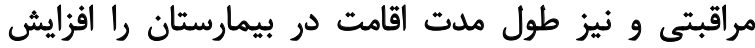

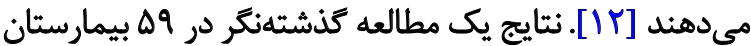

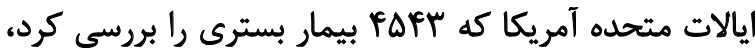

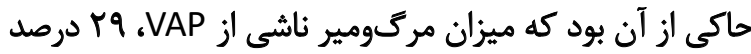

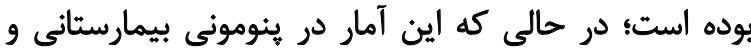

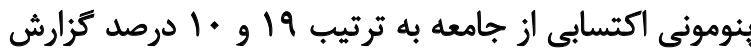

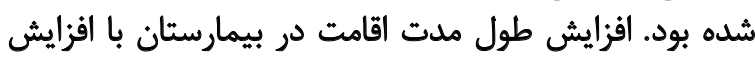

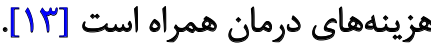

در يك بررسى، تعداد موارد يُنومونى بيمارستانى در بيمارانى ئي

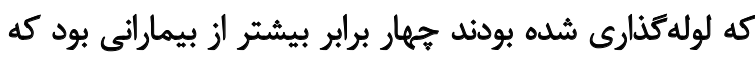

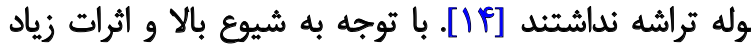

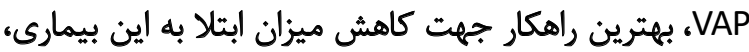

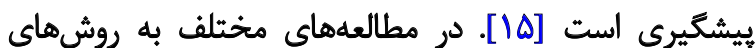

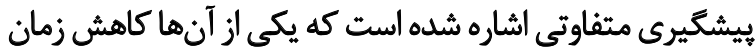

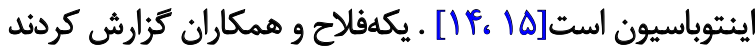

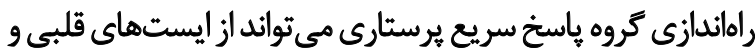

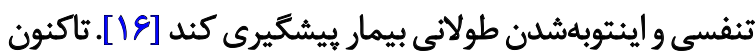

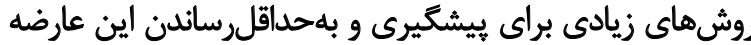

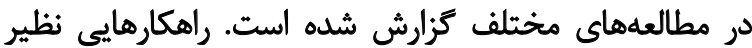

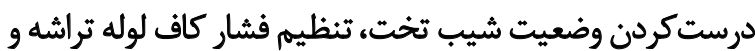

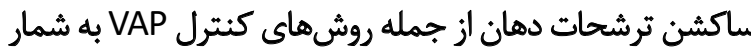

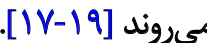

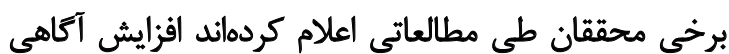

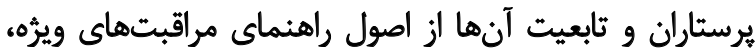


بيوتيكها، ميزان شيوع تراكئوبرونشيت وابسته به دستكاه كه

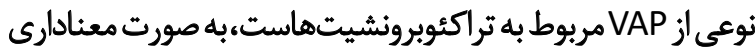

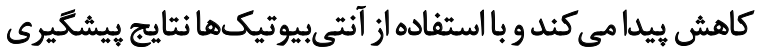

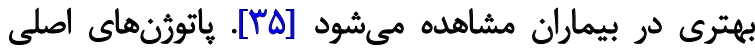

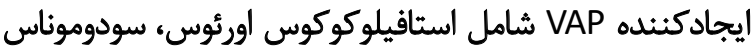

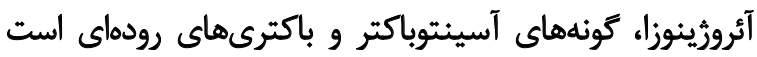

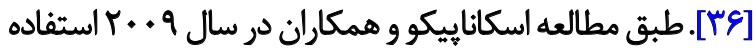

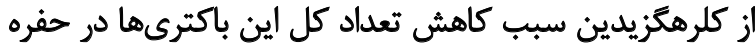
دهان نمىشود؛ اما ميزان كلونى استافيلوكوكوس اورئ تعائوس

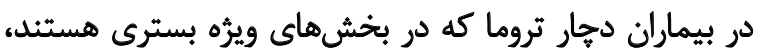

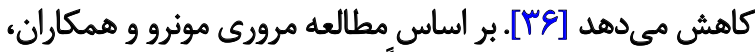

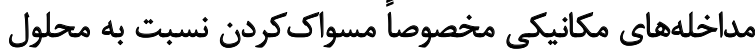

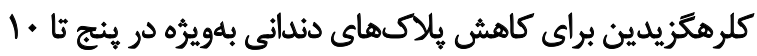

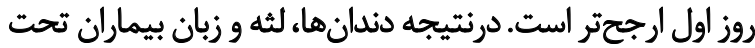

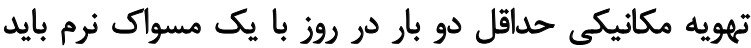
مسواك شود [TrV].

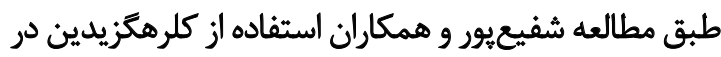

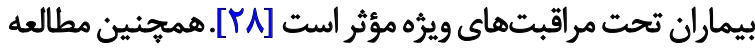

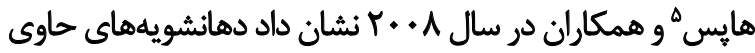

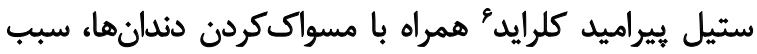

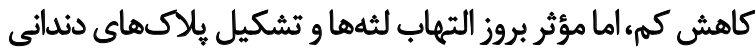

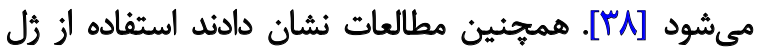

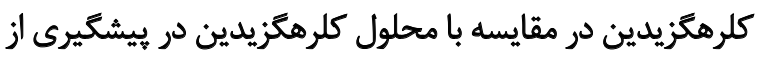

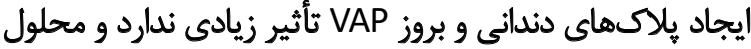

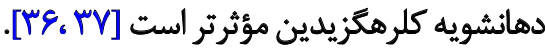

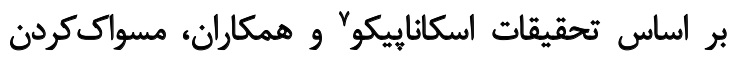

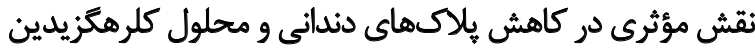
اثر زيادى در كاهش ميكروبهائ حلق دائق و ايجاد

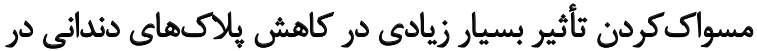

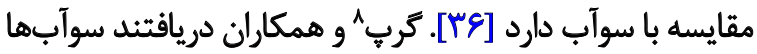

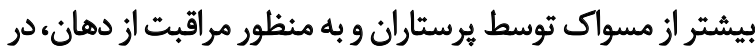

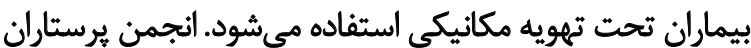

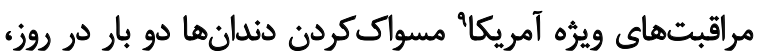

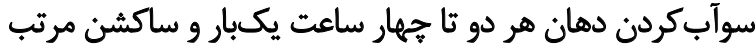

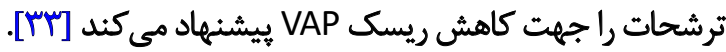
البته در مورد بهداشت دهان و مسواك مطالعات متناقضى

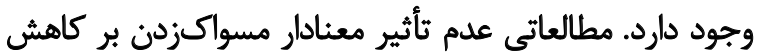

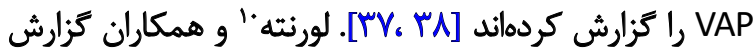

4. Associated tracheobronchitis

6. cetylpyridinium chloride

7. Scannapieco

8. Grap

9. American association of critical care nurses (ACCN)

10. Lorente
منتشر شده بود، جستوجو شدند. كليدوارههاي استفادهده

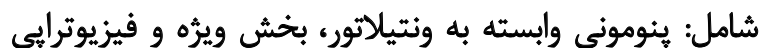
قفسه سينه بود. تينومون.

معيارهاي انتخاب مقالات شامل اين مواردبود: مقالات يافتشده

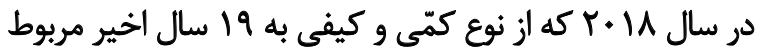

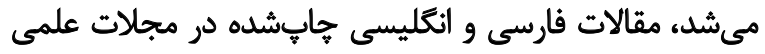

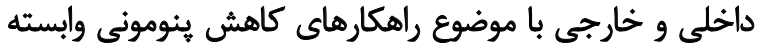

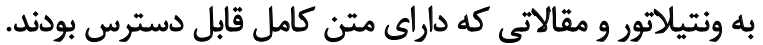

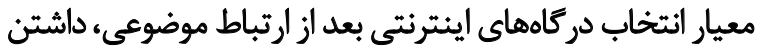

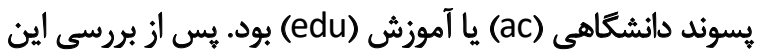

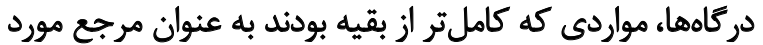

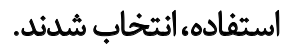
از موتور جستوجوى كوكل براى اطمينان از جستوجو

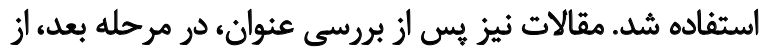

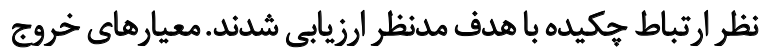

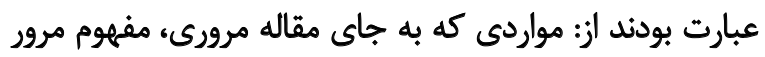

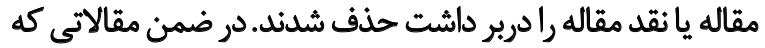
نحوه اجراى آنها نامشخص و متن كامل آنها آنها غيرقابل دسترس

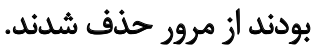
مراحل انجام كار به اين ترتيب بود كه يك نفر از محققان

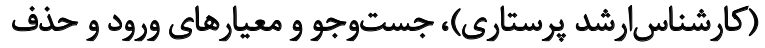

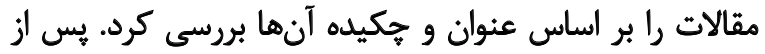

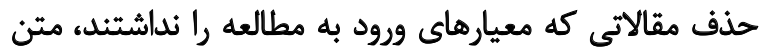

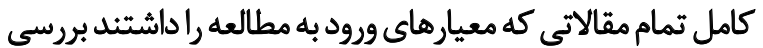

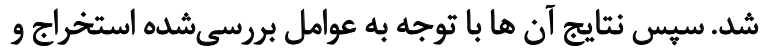

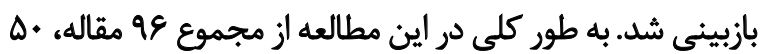

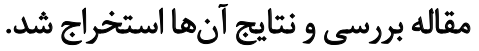

\section{بحث و وتئهان كَيرى}

وجود لوله تراشه و نيز بهداشت ضعيف دهان منجر به بروز

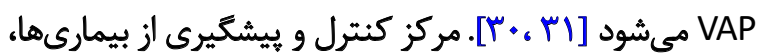

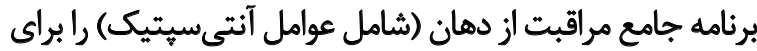

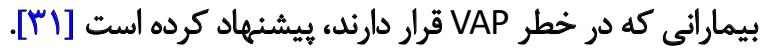

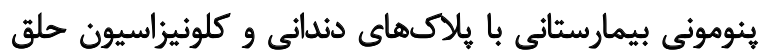

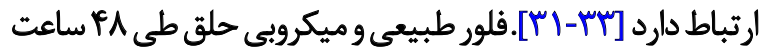

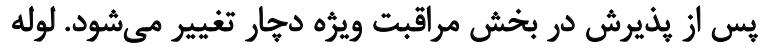

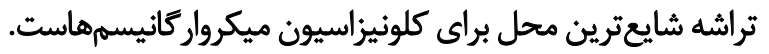

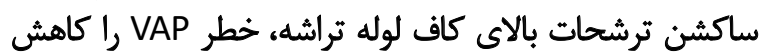

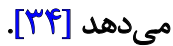

مداخلههاى دارويي نيز ثأثير زيادى در كاهش ميكروبهاى

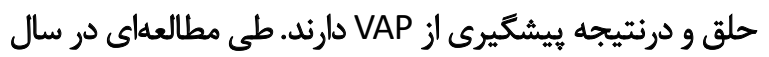

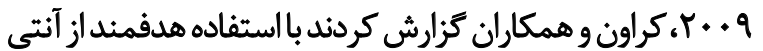




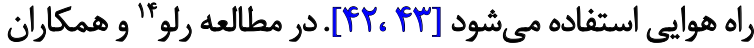

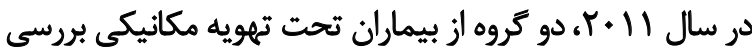

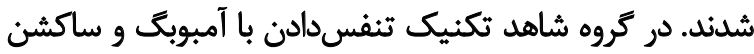

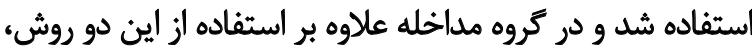

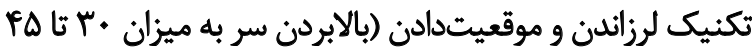

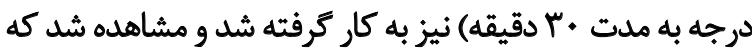

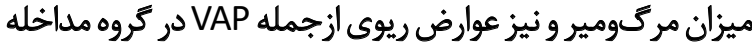

به ميزان قابل توجهى كاهش يافته بود [1/] ].

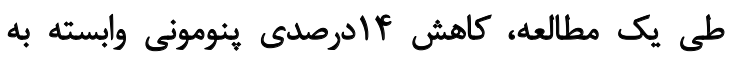
ونتيلاتور در اثر انجام مراقبتهاى استاندارد راه هوائى شامل

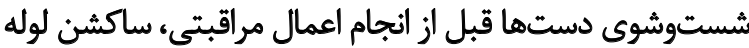

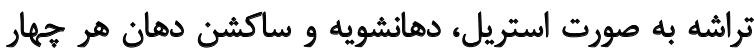

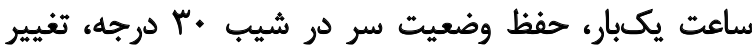

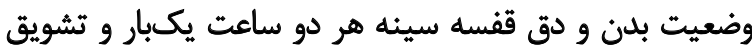

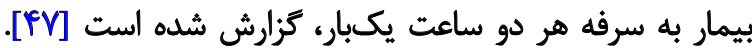

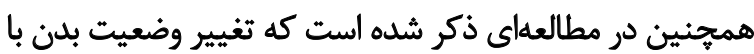

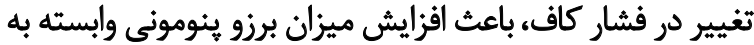

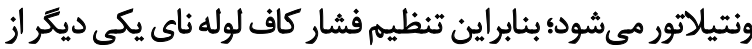

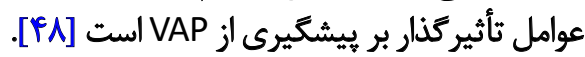

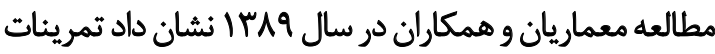

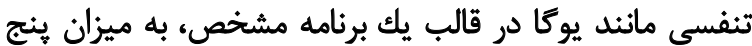

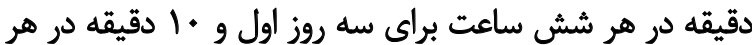

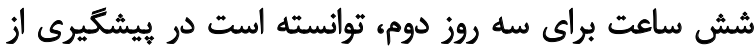

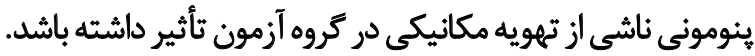

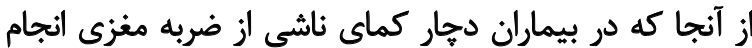

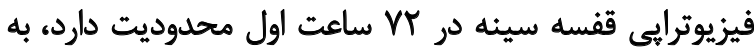

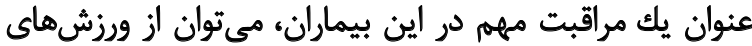

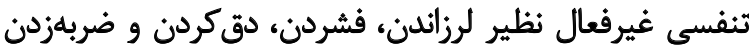

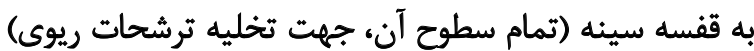

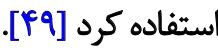

صفدره و و همكاران طى مطالعهاى اقدامات بيشكيرانه براى

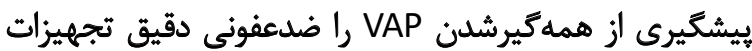

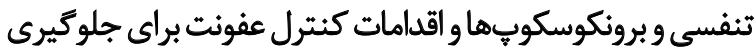

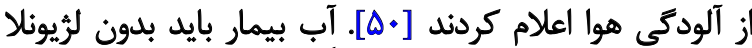

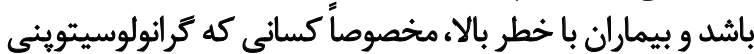

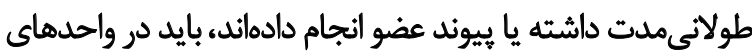

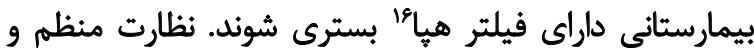

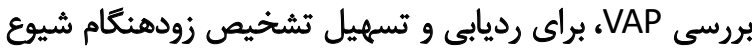

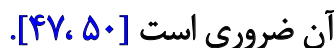

كردند مسواكزدن باعث كاهش سٓا درصدى VAP شد؛ ولى

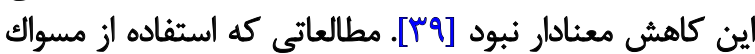

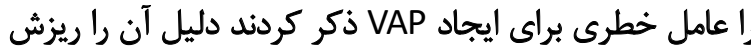

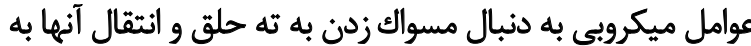

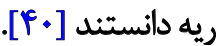

علاوه بر درمان آنتىبيوتيكى، روشهاي سادالى جهت

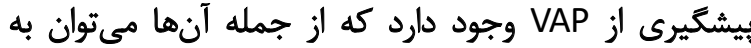

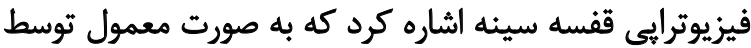

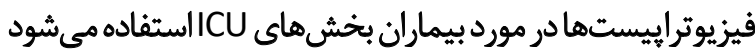

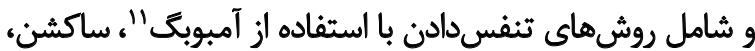

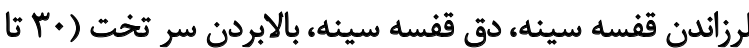
fo

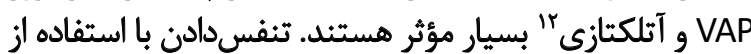

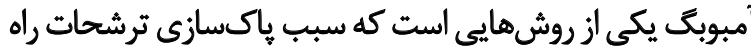

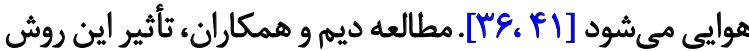

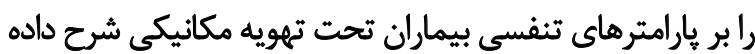

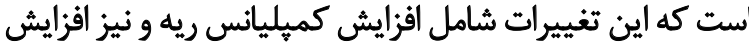

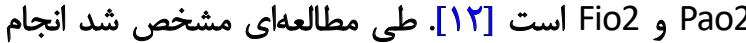

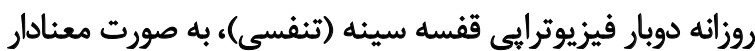

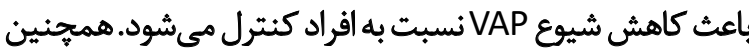
انجام اين فيزيوترايى باعث كاهش نرخ مركتومير در اين افراد نيز

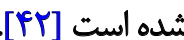

ساكشن مؤثر نيز نقش بسيار مؤثرى در بيشكيرى از بروز

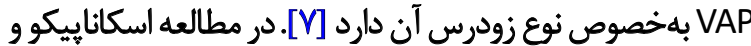

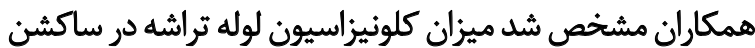

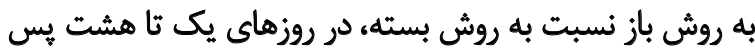

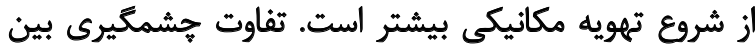

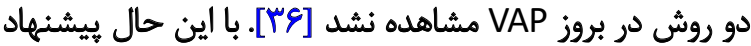

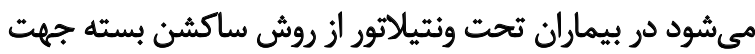

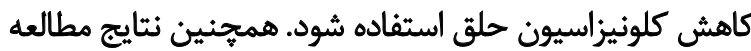

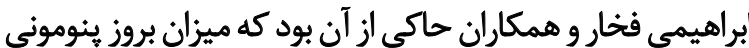

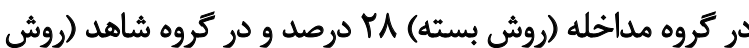

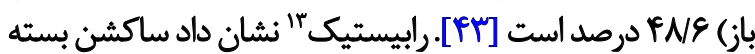

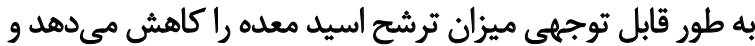

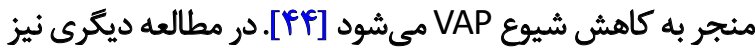

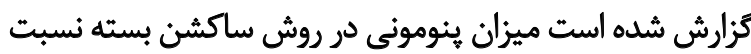

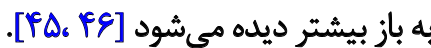
لرزاندن يكى از روشهايى است كه به روش دستى انجام

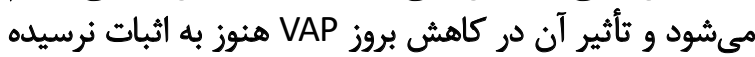

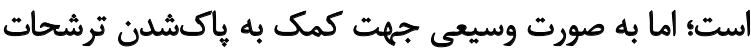


Y. موقعيت بالابردن سر تخت به ميزان FA درجه Iآ؛

r. موقعيت خوابيده به شكم.

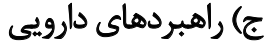

1.استفاده از يروفيلاكسى استرس اولسر؛ شواهد نشان مي دهد

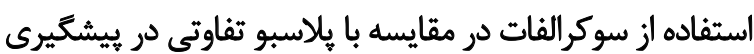

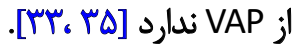

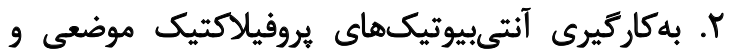
وريدى با كاهش ميزان مركومير و بروز لهمير لهراه بوده است.

كرجه ينومونى ناشى از ونتيلاتور يك عفونت شايع بيمارستانى

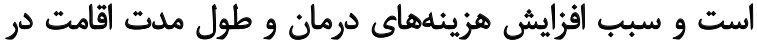

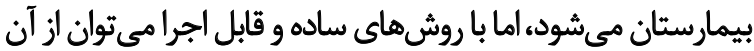

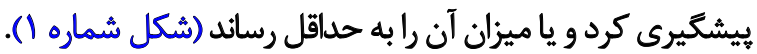

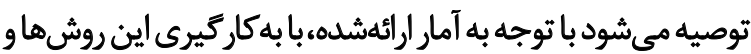

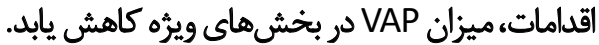

\section{مالاحظات أخلاقي \\ يبروى أز اصول الخالاق يُؤشش}

1. اصول علمى و ادبى در نتارش مقاله رعايت شده است؟؟.

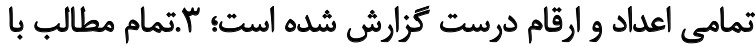
ذكر دقيق منبع و نام نويسندكان آورده شده است.

21. Semi-recumbent
دودى "ا در يك مقاله مرورى سيستماتيك با بررسي مقالات

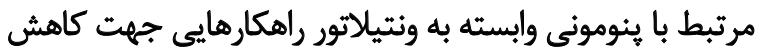

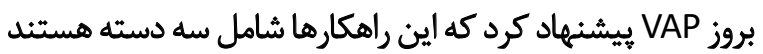

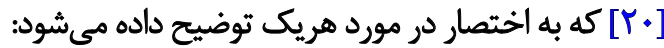

$$
\text { الف) راهبردهاى فيزيكى كه عبارتاند از: }
$$

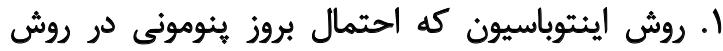

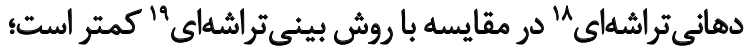
ك. دفعات تعويض تجهيزات ونتيلاتور كه بررسىها نشان داد

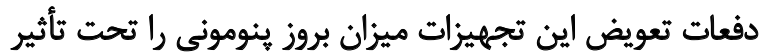

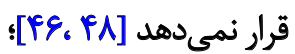
r. استفاده از هموديفاير كرم و مرطوب و نيز كاهش دفعات

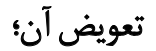
f. ساكشن بسته تراشه و تعويض آن براى هر بيمار؛ ه. ساكشن ترشحات زير كلوت؛

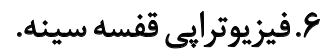

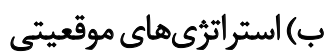

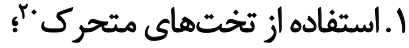

\section{Dodek}

18. Orotraceal

19. Nasotracheal

20. Kinetic

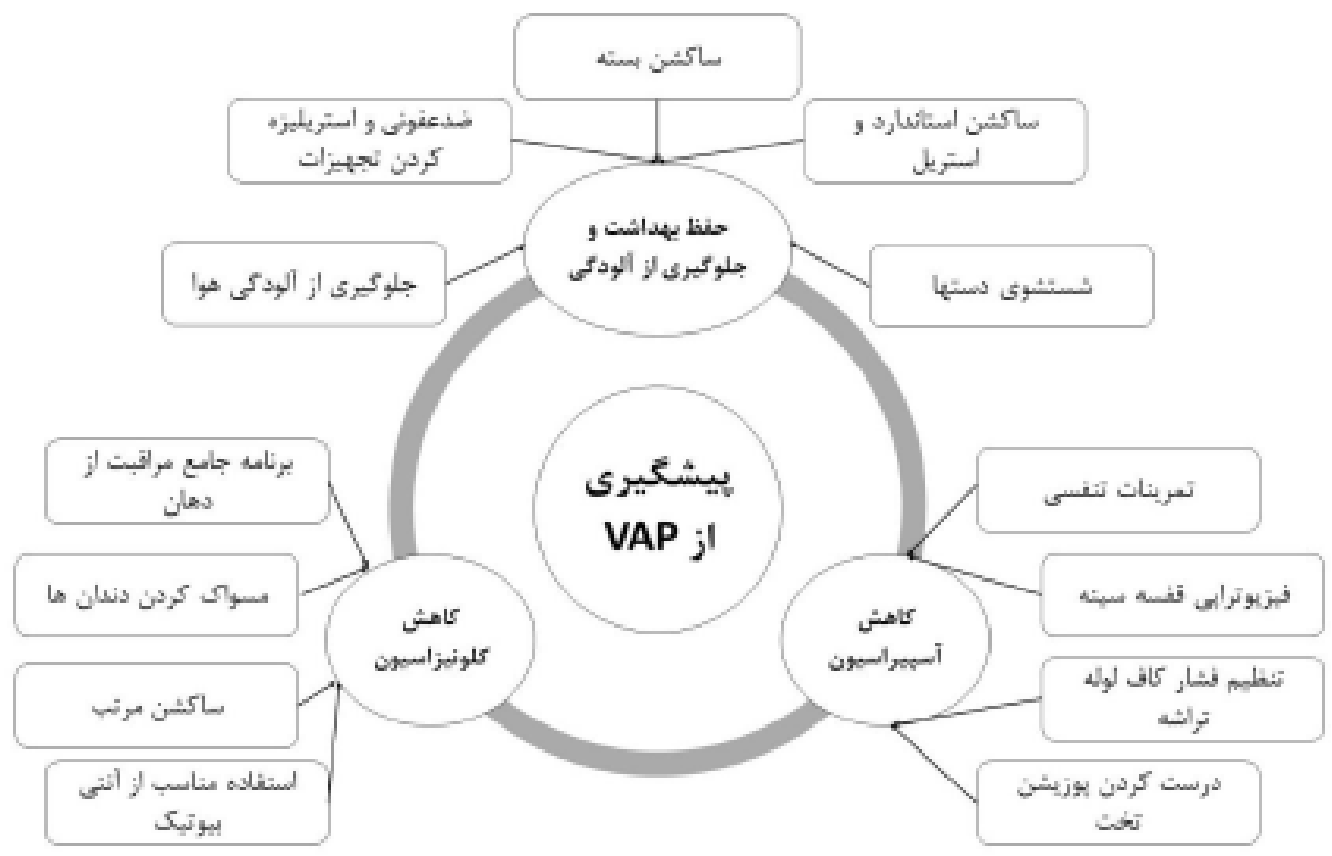




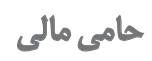

اين تحقيق هيج كمك مالى خاصى از سازمان هاى تأمين مالى در بخشهاى دولتى، تجارى يا غيرانتفاعى دريافت نكرده است

$$
\text { مشاركت نويسندكّان }
$$

جستوجو و جمعآورى منابع: محيا شفائى و ليلى يكهفلاح؛

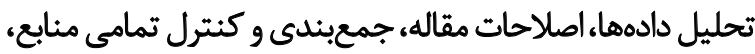

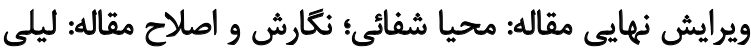

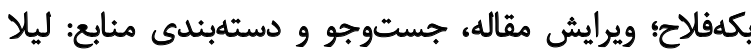
دهقانكار.

$$
\text { تقارض مثأع }
$$

در اين مطالعه هيج تونه تعارض منافعى وجود ندارد. 


\section{References}

[1] Suri HS, Li G, Gajic O. Yearbook of intensive care and emergency medicine. Berlin: Springer; 2008

[2] Rocha LD, Vilela CA, Cezário RC, Almeida AB, Gontijo Filho P. Ventilator-associated pneumonia in an adult clinical-surgical intensive care unit of a Brazilian university hospital: Incidence, risk factors, etiology, and antibiotic resistance. Braz J Infect Dis. 2008; 12(1):80-5. [DOI:10.1590/S1413-86702008000100017] [PMID]

[3] Perrie H, Scribante J. A survey of oral care practices in South African intensive care units. S Afr Med J. 2011; 27(2):42-6.

[4] Japoni A, Vazin A, Davarpanah MA, Ardakani MA, Alborzi A, Japoni $S$, et al. Ventilator-associated pneumonia in Iranian intensive care units. J Infect Dev Ctries. 2011; 5(4):286-93. [DOI:10.3855/ jidc.1212] [PMID]

[5] Efrati S, Deutsch I, Antonelli M, Hockey PM, Rozenblum R, Gurman GM. Ventilator-associated pneumonia: Current status and future recommendations. J Clin Monit Comput. 2010; 24(2):1618. [DOI:10.1007/s10877-010-9228-2] [PMID]

[6] Sadegyan E. Nosocominal management in intensive care unit. J Arak Facul Nurs Midwifery. 2005; 6:37-43. [In Persian]

[7] Olaechea PM, Ulibarrena MA, Alvarez-Lerma F, Insausti J, Palomar M, De la Cal MA, et al. Factors related to hospital stay among patients with nosocomial infection acquired in the intensive care unit. Infect Control Hosp Epidemiol. 2003; 24(3):207-13. [DOI:10.1086/502191] [PMID]

[8] Shariatpanahi ZV, Taleban FA, Mokhtari M, Shahbazi S. Ginger extract reduces delayed gastric emptying and nosocomial pneumonia in adult respiratory distress syndrome patients hospitalized in an intensive care unit. J Crit Care. 2010; 25(4):647-50. [DOI:10.1016/j.jcrc.2009.12.008] [PMID]

[9] Rosenthal VD, Maki DG, Jamulitrat S, Medeiros EA, Todi SK, Gomez DY, et al. International Nosocomial Infection Control Consortium (INICC) report, data summary for 2003-2008, issued June 2009. Am J Infect Control. 2010; 38(2):95-104. [DOI:10.1016/j. ajic.2009.12.004] [PMID]

[10] Dicocco JM, Croce MA. Ventilator-associated pneumonia: An overview. Expert Opin Pharmacother. 2010; 10(9):1461-7. [DOI:10.1517/14656560903007922] [PMID]

[11] Melsen WG, Rovers MM, Bonten MJ. Ventilator-associated pneumonia and mortality: A systematic review of observational studies. Crit Care Med. 2013; 37(10):2709-18. [In Persian] [DOI:10.1097/00003246-200910000-00005] [PMID]

[12] Deem S, Treggiari MM. New endotracheal tubes designed to prevent ventilator-associated pneumonia: Do they make a difference? Rep Care. 2010; 55(8):1046-55. [PMID]

[13] Kollef MH, Shorr A, Tabak YP, Gupta V, Liu LZ, Johannes RS. Epidemiology and outcomes of health-care-associated pneumonia: Results from a large US database of culture-positive pneumonia. Chest. 2005; 128(6):3854-62. [DOI:10.1378/chest.128.6.3854] [PMID]

[14] Kasper A, Bere N. Harrison's Principles of Internal Medicine. [F Ghorbani, A Ahmadi, P Bashiri, Persian Trans.]. Tehran: Teymurzade Publications; 2005.
[15] Hutchins K, Karras G, Erwin J, Sullivan KL. Ventilator-associated pneumonia and oral care: A successful quality improvement project. Am J Infect Control. 2009; 37(7):590-7. [DOI:10.1016/j. ajic.2008.12.007] [PMID]

[16] YekeFallah L, Eskandari Z, Shahrokhi A, Javadi A. Effect of rapid response nursing team on outcome of patient care. Trauma Mon. 2018; 23(4):e14488. [DOI:10.5812/traumamon.14488]

[17] Drakulovic MB, Torres A, Bauer TT, Nicolas JM, NoguéS, Ferrer M. Supine body position as a risk factor for nosocomial pneumonia in mechanically ventilated patients: A randomised trial. Lancet. 1999; 354(9193):1851-8. [DOI:10.1016/S0140-6736(98)12251-1]

[18] Rello J, Sonora R, Jubert P, Artigas A, Rué M, Vallés J. Pneumonia in intubated patients: Role of respiratory airway care. Am J Respir Crit Care Med. 1996; 154(1):111-5. [DOI:10.1164/ajrccm.154.1.8680665] [PMID]

[19] Chao YF, Chen YY, Wang KW, Lee RP, Tsai H. Removal of oral secretion prior to position change can reduce the incidence of ventilator-associated pneumonia for adult ICU patients: A clinical controlled trial study. J Cli Nurs. 2009; 18(1):22-8. [DOI:10.1111/ j.1365-2702.2007.02193.x] [PMID]

[20] Dodek P, Keenan S, Cook D, Heyland D, Jacka M, Hand L, et al. Evidence-based clinical practice guideline for the prevention of ventilator-associated pneumonia. Ann Intern Med. 2004; 141(4):30513. [DOI:10.7326/0003-4819-141-4-200408170-00011] [PMID]

[21] Muscedere J, Dodek P, Keenan S, Fowler R, Cook D, Heyland D, et al. Comprehensive evidence-based clinical practice guidelines for ventilator-associated pneumonia: Prevention. J Critic Care. 2008; 23(1):126-37. [DOI:10.1016/j.jcrc.2007.11.014]

[22] Gallagher JA. Implementation of ventilator-associated pneumonia clinical guideline (bundle). J Nurse Pract. 2012; 8(5):377-82. [DOI:10.1016/j.nurpra.2012.02.017]

[23] Svatka M, Nihada B, Natalie J, Matilda H, Stephen H, Hayley B. Reducing risk for ventilator associated pneumonia through nursing sensitive interventions. Intensive Crit Care Nurs. 2013; 29(5): 261-5. [DOI:10.1016/j.iccn.2013.04.005] [PMID]

[24] Blot SI, Labeau S, Vandijck D, Van Aken P, Claes B, Executive Board of the Flemish Society for Critical Care Nurses. Evidencebased guidelines for the prevention of ventilator-associated pneumonia: Results of a knowledge test among intensive care nurses. Intensive Care Med. 2007; 33(8):1463-7. [DOI:10.1007/ s00134-007-0705-0] [PMID]

[25] El-Khatib MF, Zeineldine S, Ayoub C, Husari A, Bou-Khalil PK. Critical care clinicians' knowledge of evidence-based guidelines for preventing ventilator-associated pneumonia. Am J Crit Care. 2010; 19(3):272-6. [DOI:10.4037/ajcc2009131] [PMID]

[26] Cocanour CS1, Peninger M, Domonoske BD, Li T, Wright B, Valdivia $A$, et al. Decreasing ventilator-associated pneumonia in a trauma ICU. J Trauma Acute Care Surg. 2006; 61(1):122-30. [DOI:10.1097/01.ta.0000223971.25845.b3] [PMID]

[27] El Azab SR, El Sayed AE, Abdelkarim M, Al Mutairi KB, Al Saqabi A, El Demerdash S. Combination of ventilator care bundle and regular oral care with chlorhexidine was associated with reduction in ventilator associated pneumonia. Egypt J Anaesth. 2013 29(3):273-7. [DOI:10.1016/j.egja.2013.03.001] 
[28] Shafipour V, Haghighi A, Bagheri nesami M. The impact of oral care in the prevention of ventilator-associated pneumonia: $A$ narrative review. Iran J Nurs Res. 2018; 12(6):25-36. [In Persian] [DOI:10.21859/ijnr-12064]

[29] Ross A, Crumpler J. The impact of an evidence-based practice education program on the role of oral care in the prevention of ventilator-associated pneumonia. Intensive Crit Care Nurs. 2007; 23(3):132-6. [DOI:10.1016/j.iccn.2006.11.006] [PMID]

[30] Chen G, Wang J, Liu C, Xu R, Li Q, Zhou X, et al. Subglottic secretion drainage and semi-recumbent position for preventing ventilator associated pneumonia. Int J Clin Exp Med. 2016; 9(2):51938.

[31] El-Solh AA, Pietrantoni C, Bhat A, Okada M, Zambon J, Aquilina $A$, et al. Colonization of dental plaques: A reservoir of respiratory pathogens for hospital-acquired pneumonia in institutionalized elders. Chest. 2004; 126(5):1575-82. [DOI:10.1016/S00123692(15)31374-X]

[32] Fourrier F, Dubois D, Pronnier P, Herbecq P, Leroy O, Desmettre $T$, et al. Effect of gingival and dental plaque antiseptic decontamination on nosocomial infections acquired in the intensive care unit: A double-blind placebo-controlled multicenter study. Crit Care Med. 2005; 33(8):1728-35. [DOI:10.1097/01. CCM.0000171537.03493.BO] [PMID]

[33] Grap MJ, Munro CL, Elswick Jr RK, Sessler CN, Ward KR. Duration of action of a single, early oral application of chlorhexidine on oral microbial flora in mechanically ventilated patients: A pilot study. Heart Lung. 2004; 33(2):83-91. [DOI:10.1016/j. hrtlng.2003.12.004] [PMID]

[34] Combes P, Fauvage B, Oleyer C. Nosocomial pneumonia in mechanically ventilated patients, a prospective randomised evaluation of the Stericath closed suctioning system. Intensive Care Med. 2000; 26(7):878-82. [DOI:10.1007/s001340051276] [PMID]

[35] Craven DE, Chroneou A, Zias N, Hjalmarson KI. Ventilator-associated tracheobronchitis: The impact of targeted antibiotic therapy on patient outcomes. Chest. 2009; 135(2):521-8. [DOI:10.1378/ chest.08-1617] [PMID]

[36] Scannapieco FA, YU J, Raghavendran K, Vacanti A, Owens SI, Wood K, et al. A randomized trial of chlorhexidine gluconate on oral bacterial pathogens in mechanically ventilated patients. Crit Care. 2009; 13(4):1-12. [DOI:10.1186/cc7967] [PMID] [PMCID]

[37] Munro CL, Grap MJ, Jones DJ, McClish DK, Sessler CN. Chlorhexidine, toothbrushing, and preventing ventilator-associated pneumonia in critically ill adults. Am J Crit Care. 2009; 18(5):428-37. [DOI:10.4037/ajcc2009792] [PMID] [PMCID]

[38] Haps S, Slot DE, Berchier CE, Van der Weijden GA. The effect of cetylpyridinium chloride-containing mouth rinses as adjuncts to toothbrushing on plaque and parameters of gingival inflammation: A systematic review. Int J Dent Hygiene. 2008; 6(4):290-303. [DOI:10.1111/j.1601-5037.2008.00344.x] [PMID]

[39] Lorente L, Lecuona M, Jiménez A, Palmero S, Pastor E, Lafuente $\mathrm{N}$, et al. Ventilator-associated pneumonia with or without toothbrushing: A randomized controlled trial. Eur J Cli Microbiol Infect Dis. 2012; 31(10):2621-9. [DOI:10.1007/s10096-0121605-y] [PMID]
[40] Pattanshetty RB, Gaude GS. Effect of multimodality chest physiotherapy in prevention of ventilator-associated pneumonia: $A$ randomized clinical trial. Indian J Crit Care Med. 2010; 14(2):70-6. [DOI:10.4103/0972-5229.68218] [PMID] [PMCID]

[41] Alhazzani W, Smith O, Muscedere J, Medd J, Cook D. Toothbrushing for critically ill mechanically ventilated patients: A systematic review and meta-analysis of randomized trials evaluating ventilator-associated pneumonia. Crit Care Med. 2013; 41(2):646-55. [DOI:10.1097/CCM.0b013e3182742d45] [PMID]

[42] Ntoumenopoulos G, Presneill JJ, McElholum M, Cade JF. Chest physiotherapy for the prevention of ventilator-associated pneumonia. Intensive Care Med. 2002; 28(7):850-6. [DOI:10.1007/ s00134-002-1342-2] [PMID]

[43] Ebrahimi Fakhar H, Rezaei K, Kohistani H R. Effect of suction of lung secretion on ventilator-associated- pneumonia. Sci J Kurdis$\tan$ Uni Med Sci. 2010; 15(2):79-87. [In Persian]

[44] Rabitsch W, Köstler WJ, Fiebiger W, Dielacher C, Losert H, Sherif $\mathrm{C}$, et al. Closed suctioning system reduces cross-contamination between bronchial system and gastric juices. Anaesth Analg . 2004; 99(3):886-92. [DOI:10.1213/01.ANE.0000143353.85428.39] [PMID]

[45] Lee ES, Kim SH, Kim JS. Effects of a closed endotracheal suction system on oxygen saturation, ventilator-associated pneumonia, and nursing efficacy. J Korean Acad Nurs. 2004; 34(7):1315-25 [DOI:10.4040/jkan.2004.34.7.1315] [PMID]

[46] Salimi SA, Nowroo S, Anami I, Akdemir N, Rastat MA. Effect of standardization of nursing cares on incidence of nosocomial infection in MICU. J Urmia Univ Med Sci. 2009; 19(4):310-5. [PMID] [PMCID]

[47] Shahrokhi A, Mohammadi Alamoti A, YekeFallah L, Mohammad Pouraslash $A$. The effect of body position change on the pressure of the oral cavity tube cuff in patients with mechanical ventilation. J Qazvin Univ Med Sci. 2010; 13(5):26-33. [PMID] [PMCID]

[48] Kirton OC, DeHaven B, Morgan J, Morejon O, Civetta J. A prospective, randomized comparison of an in-line heat moisture exchange filter and heated wire humidifiers: rates of ventilatorassociated early-onset (community-acquired) or late-onset (hospital-acquired) pneumonia and incidence of endotracheal tube occlusion. Chest. 1997; 112(4):1055-9. [DOI:10.1378/ chest.112.4.1055] [PMID]

[49] Memarian R, Misaghi A, Brujerdi M, Nazem ekbatani N. Effect of planned passive respiratory exercise on prevention of pneumonia among coma patients undergoing mechanical ventilation. Hayat. 2010; 16(2):29-37. [In Persian]

[50] Safdar N, Crnich CJ, Maki DG. The pathogenesis of ventilator-associated pneumonia: Its relevance to developing effective strategies for prevention. Respir Care. 2005; 50(6):725-41. [PMID] 
This Page Intentionally Left Blank 